\title{
ARTICLE
}

Received 16 Oct 2014 | Accepted 25 Jul 2015 | Published 4 Sep $2015 \quad$ DOl: 10.1038/ncomms9172

\section{Imaging of alignment and structural changes of carbon disulfide molecules using ultrafast electron diffraction}

Jie Yang ${ }^{1}$, Joshua Beck ${ }^{1}$, Cornelis J. Uiterwaal ${ }^{1} \&$ Martin Centurion ${ }^{1}$

Imaging the structure of molecules in transient-excited states remains a challenge due to the extreme requirements for spatial and temporal resolution. Ultrafast electron diffraction from aligned molecules provides atomic resolution and allows for the retrieval of structural information without the need to rely on theoretical models. Here we use ultrafast electron diffraction from aligned molecules and femtosecond laser mass spectrometry to investigate the dynamics in carbon disulfide following the interaction with an intense femtosecond laser pulse. We observe that the degree of alignment reaches an upper limit at laser intensities below the ionization threshold, and find evidence of structural deformation, dissociation and ionization at higher laser intensities.

\footnotetext{
${ }^{1}$ Department of Physics and Astronomy, University of Nebraska-Lincoln, Lincoln, Nebraska 68588, USA. Correspondence and requests for materials should be addressed to M.C. (email: martin.centurion@unl.edu).
} 
aser alignment of molecules has been proposed as a method to achieve three-dimensional diffractive imaging ${ }^{1-3}$. Recent experiments have shown that it is possible to record electron ${ }^{4}$ and X-ray ${ }^{5}$ diffraction patterns of impulsively aligned molecules, and to retrieve the molecular structure with atomic resolution ${ }^{6}$. Impulsive alignment is often preferable to adiabatic alignment, because the molecules can be imaged in a field-free environment. The degree of alignment improves with increasing laser intensity, however, at high intensities the alignment laser pulse can cause structural deformation and ionization of the molecules. To determine the intensity threshold at which these changes appear, it is necessary to simultaneously determine the probability of ionization, the degree of alignment and the structure of the excited molecules. This is important to ensure that molecular imaging experiments are carried out at intensity levels that do not distort the structure.

The alignment, electronic excitation and dissociation dynamics of carbon disulfide $\left(\mathrm{CS}_{2}\right)$ have drawn much recent interest ${ }^{7-16}$. Although being one of the simplest polyatomic molecules, $\mathrm{CS}_{2}$ shares many general and important properties with other polyatomic molecules such as the presence of conical intersections and the ability to be photoexcited into dissociative states. Laser-based femtosecond pump-probe experiments have been a powerful method for studying molecular dynamics in the gas phase. A probe femtosecond laser pulse, for example, can ionize the molecule, and the resulting fragments (electrons and ions) studied using kinetic-energy-resolved velocity map imaging $^{17,18}$. Diffraction techniques, on the other hand, provide direct structural information of molecules. X-Ray diffraction using an X-Rayfree electron laser could be used to observe structural changes with angstrom spatial resolution, provided sub-angstrom wavelength X-Ray pulses with high fluence become available $^{19}$. Ultrafast electron diffraction (UED) has been used to measure conformational changes in molecules in the gas phase with a temporal resolution of a few picoseconds ${ }^{4,20,21}$, and UED from aligned molecules (UEDAM) has been used to measure three-dimensional static molecular structures ${ }^{6}$.

In this work, we use UEDAM to measure the degree of alignment and structure of molecules after excitation with an intense $\left(10^{12}-3 \times 10^{13} \mathrm{~W} \mathrm{~cm}^{-2}\right)$ femtosecond infrared laser pulse, and femtosecond laser mass spectrometry (FLMS) to measure the degree of ionization. $\mathrm{CS}_{2}$ is chosen as a model system for this study. UEDAM allows us to retrieve both the transient molecular structure with atomic spatial resolution and the angular distribution of the molecular ensemble directly from the data without relying on theoretical modelling. We use FLMS to accurately determine the ionization products and their population as a function of intensity. Laser pulses with intensities in the range of $10^{12}-10^{14} \mathrm{~W} \mathrm{~cm}^{-2}$ are often used to align molecules or to probe excited molecules by multiphoton ionization ${ }^{7,8,12}$, but their influence on the molecular structure has not been fully explored. These high intensities excite a broad range of dynamical phenomena, including excitation of rotational, vibrational and electronic states leading to alignment, deformation, dissociation and ionization. With UEDAM and FLMS, we find evidences of alignment, deformation, dissociation and ionization at different levels of laser intensity.

\section{Results}

Experimental setup and diffraction pattern. The schematic for the UEDAM experimental setup is shown in Fig. 1a. A single laser pulse with a wavelength of $800 \mathrm{~nm}$ is used to excite the molecules, and a femtosecond electron pulse is used to record diffraction patterns for a range of time delays with respect to the laser pulse. The electron beam, laser beam and gas jet are mutually orthogonal. The overall temporal resolution of the experiment is $1.0 \mathrm{ps}$ and is determined by the duration of the laser and electron pulses and the transverse width of the electron, laser and gas beams. The anisotropy of the diffraction pattern is used to monitor the degree of alignment (Fig. 1b). The anisotropy as a function of time is measured for each value of the laser intensity. After finding the time delay corresponding to the alignment maximum closest to zero delay, $\sim 10^{9}$ scattering events are accumulated to record a diffraction pattern with a high signal-to-noise ratio. The ionization products and the fraction of ionized molecules were measured using FLMS. An ultrashort laser pulse ( $50 \mathrm{fs}$ duration, $800 \mathrm{~nm}$ wavelength) is focused into a dilute $\mathrm{CS}_{2}$ gas $\left(\sim 10^{-7}\right.$ mbar $)$ and ion yields are recorded as a function of pulse energy using a time-of-flight ion mass spectrometer. The spectrometer has a fixed, micrometer-sized interaction volume that is smaller than the focal volume of the laser $^{22}$. Thus, the target is a fixed number of molecules and the yields are not averaged over the spatial intensity profile of the laser focus. Further experimental details may be found in refs 22-25. The inverse Fourier transform of the diffraction pattern intensity $I$ is the autocorrelation function of the molecular structure convolved with its angular distribution (see the Molecular images section in Methods section). For a linear molecule that is aligned perfectly, an image of the structure can be retrieved directly from the autocorrelation function. Figure 1c,d show a simulation of the diffraction pattern $I$ and its inverse Fourier transform for perfectly aligned $\mathrm{CS}_{2}$ molecules. The two bright spots in Fig. 1d represent the C-S and S-S distances in the $\mathrm{CS}_{2}$ molecule, as shown by the two white arrows. The spot at the centre of the image, which contains no structural information, was removed for clarity. Figure 1e displays a model of the $\mathrm{CS}_{2}$ molecule with $\mathrm{C}-\mathrm{S}$ and $\mathrm{S}-\mathrm{S}$ distances marked for comparison. If the alignment is not perfect, the inverse Fourier transform gives a two-dimensional projection of the three-dimensional image of the molecular ensemble, or equivalently an Abel transform of all the molecules along the direction of electron propagation.

Saturation of alignment and ionization. The maximum degree of alignment that can be reached by impulsive excitation, in the limit of very short pulses, depends on the laser fluence (rather than intensity) ${ }^{26}$. For example, two very short pulses with different pulse durations and the same energy will produce the same alignment. Other processes, such as multiphoton excitation and ionization depend strongly on the laser intensity. We have investigated the dynamics both as a function of fluence and intensity, by using two different pulse durations (60 and $200 \mathrm{fs}$ ) for each value of the laser pulse energy. The $200 \mathrm{fs}$ pulse is generated by chirping the $60 \mathrm{fs}$ pulse, so both pulses have the same spectrum. We have used six different laser fluences: 0.16 , $0.48,0.79,1.10,1.42$ and $1.73 \mathrm{~J} \mathrm{~cm}^{-2}$. The laser intensity ranges from $8 \times 10^{11} \mathrm{~W} \mathrm{~cm}^{-2}$ to $2.9 \times 10^{13} \mathrm{~W} \mathrm{~cm}^{-2}$. Laser ionization creates a plasma that can distort the diffraction pattern due to electric and magnetic fields induced by charge separation. The strength of the plasma fields increases with time, on a time scale of several picoseconds ${ }^{27}$. We have carefully monitored the electron beam during experiments and found that it is not distorted, except at the point with highest intensity $\left(1.73 \mathrm{~J} \mathrm{~cm}^{-2}\right.$ at $60 \mathrm{fs}$ ). This point is excluded in the following analysis, thus the diffraction patterns used in the analysis are not distorted by the plasma fields, and the maximum intensity in the UEDAM experiments is $2.4 \times 10^{13} \mathrm{~W} \mathrm{~cm}^{-2}$. The FLMS experiments have probed higher intensities to determine the values where the sample is fully ionized.The anisotropy was measured as a function of time for each value of the laser fluence and pulse duration. 
a

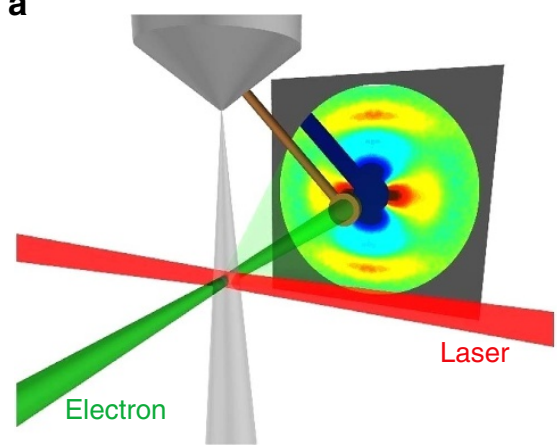

b

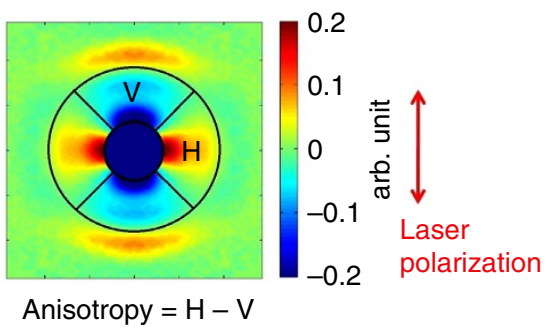

d

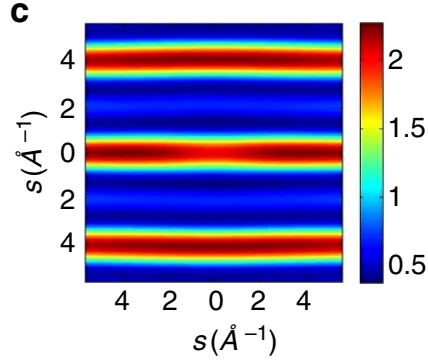

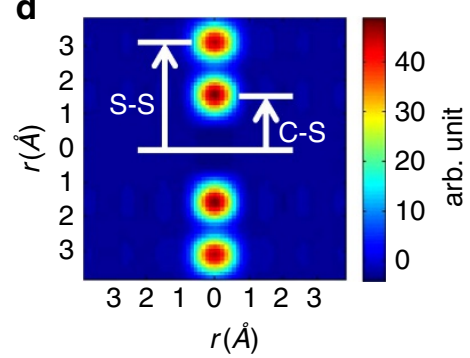

e

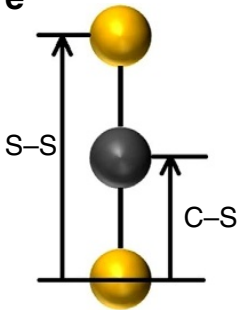

Figure 1 | Experimental setup and data analysis methods. (a) Experimental setup. The green, red and grey beams represent the electron beam, laser beam and gas jet, respectively. The beam stop is used to block the directly transmitted electrons, and the scattering pattern is recorded using a phosphor screen that is imaged on a CCD (charge-coupled device) camera. (b) The anisotropy is calculated by taking the difference of the total counts in the horizontal (marked by ' $\mathrm{H}$ ') and the vertical (marked by ' $\mathrm{V}$ ') quadrants between two circles (more details in the Molecular images section in Methods section). The diffraction patterns are normalized before calculating the anisotropy. The laser polarization is linear, shown by the red arrow. (c) Simulated diffraction pattern corresponding to perfect alignment. (d) Autocorrelation function of the molecule obtained by an inverse Fourier transform of the simulated diffraction pattern (see the Molecular images section in Methods section). Each off-centre spot represents an interatomic distance of $\mathrm{CS}_{2}$, as shown by the two arrows. (e) A ball and stick model of $\mathrm{CS}_{2}$ molecule with $\mathrm{C}-\mathrm{S}$ and $\mathrm{S}-\mathrm{S}$ distances are marked.

The delay between alignment laser pulse and the peak alignment decreases as the laser pulse intensity increases (this effect will be discussed in more detail later in this paper). The corresponding diffraction patterns are simulated for the experimental conditions (see the Alignment simulation and Diffraction pattern simulation sections in Methods section). Only rotational excitation states are included in the simulation. A significant disagreement between measurement and simulation indicates that effects other than excitation of rotational states are present.

Figure $2 \mathrm{a}$ shows the maximum anisotropy versus fluence. The peak anisotropy for each intensity is measured at the time of maximum alignment, which ranges from 1.5 to $0.9 \mathrm{ps}$ for the $200 \mathrm{fs}$ (long) laser pulses, and from $1.1 \mathrm{ps}$ to $200 \mathrm{fs}$ for the $60 \mathrm{fs}$ (short) laser pulses. The red and blue curves in Fig. 2a show the experimental and simulated results, respectively; while the solid lines represent $60 \mathrm{fs}$ pulse durations and the dashed lines represent $200 \mathrm{fs}$ pulse duration. The right axis corresponds to $\left.<\cos ^{2} \alpha\right\rangle$ values from simulation, where $\alpha$ represents the angle between the molecular axis and the laser polarization. The simulations show that the alignment by short or long laser pulses is very similar, with only a small disagreement in the highest intensities. The experimental results agree with the simulations at low fluence and saturate at around $0.8 \mathrm{~J} \mathrm{~cm}^{-2}$ for $200 \mathrm{fs}$ excitation (indicated by the roman numeral I in Fig. 2) and around $0.5 \mathrm{~J} \mathrm{~cm}^{-2}$ for $60 \mathrm{fs}$ excitation (indicated by the roman numeral III).

This measurement indicates that dynamical processes other than excitation of rotational states are present above a threshold value of intensity that is different for different laser pulse durations. The accompanying structural changes in the molecule can be determined by examining the diffraction patterns at these points, but first we examine the role of ionization. Figure $2 b$ shows the number of $\mathrm{CS}_{2}^{+}$ions and $\mathrm{CS}_{2}^{++}$ions as a function of laser intensity in log-log scale, measured using the FLMS setup. In the lower-intensity region, the data follow the expected dependence on the seventh power of the laser intensity for a seven-photon multiphoton ionization process (the ionization potential is $10.08 \mathrm{eV}$ and the photon energy is $1.55 \mathrm{eV}$ ). This region is used to fit to a seven-photon multiphoton ionization model (see FLMS Experiments and data analysis in Methods section). In the saturation region, the data underestimate ionization as compared with the model. We take the maximum number of measured ions as the $100 \%$ level of ionization for the model. Thus, the data give a lower estimate for the ionization and the model gives a higher estimate. At an intensity of $1.34 \times 10^{13} \mathrm{~W} \mathrm{~cm}^{-2}$, the model predicts $9.6 \%$ ionization, while the measurement gives a value of $7.9 \%$ ionization. For lower intensities, the model and data results are much closer to each other. At an intensity of $10^{13} \mathrm{~W} \mathrm{~cm}^{-2}, 1.3 \%$ of the molecules are ionized. For lower intensities, the number of ionized molecules decreases with the seventh power of the intensity. The fraction of $\mathrm{CS}_{2}^{++}$ions is $0.02 \%$ at an intensity of $10^{13} \mathrm{~W} \mathrm{~cm}^{-2}$ and $3.6 \%$ at a laser intensity of $2.4 \times 10^{13} \mathrm{~W} \mathrm{~cm}^{-2}$, which corresponds to the intensity at point $\mathrm{V}$ in Fig. 2a. The number of charged fragments $\left(\mathrm{S}^{+}\right.$and $\mathrm{CS}^{+}$) was also measured and was found to remain below $1 \%$ even at an intensity of $2.4 \times 10^{13} \mathrm{~W} \mathrm{~cm}^{-2}$.

Figure 2a,b together show that the saturation of alignment occurs at much lower intensity than the saturation of ionization. For alignment with the longer laser pulses ( $200 \mathrm{fs})$, the alignment reaches a saturation value at an intensity of $4 \times 10^{12} \mathrm{~W} \mathrm{~cm}^{-2}$ (point I in Fig. 2a), where the ionization is expected to be below 1 part in $10^{4}$. Ionization is thus not the mechanism that prevents the alignment from further increasing with the laser fluence. 
a
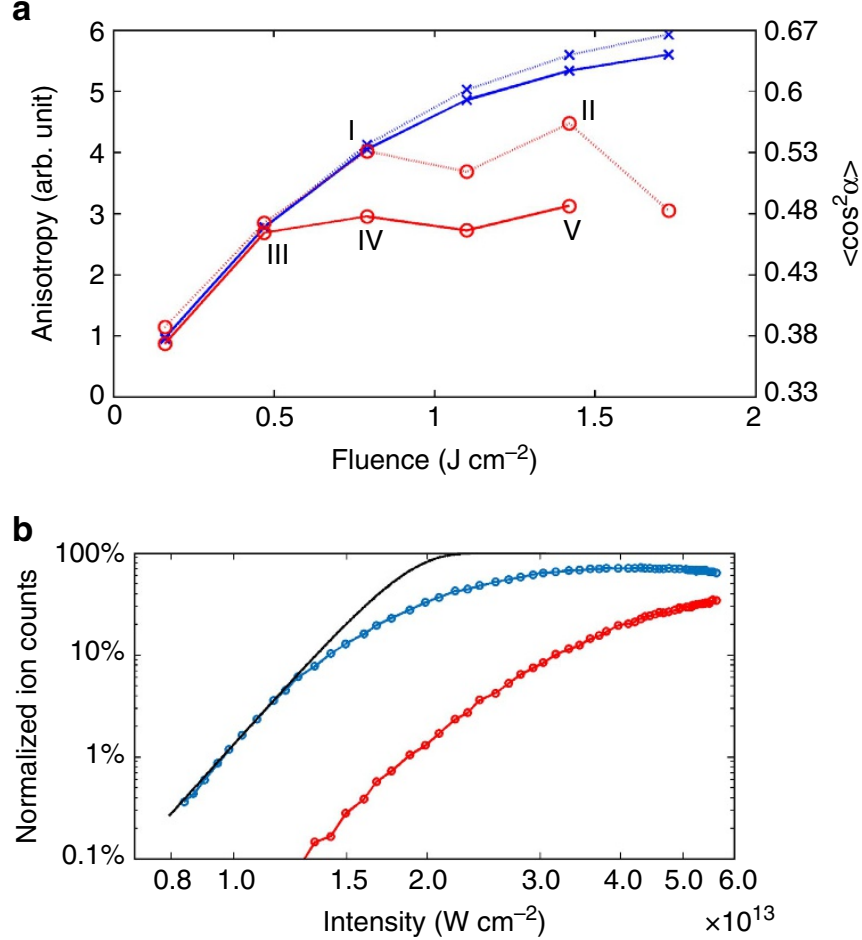

Figure 2 | Saturation of alignment and ionization. (a) Anisotropy/ alignment parameter $\left\langle\cos ^{2} \alpha\right\rangle$ versus fluence. The blue curves are simulation results, and the red curves are experimental data. The solid and dashed curves represent pulse durations of 60 and $200 \mathrm{fs}$, respectively. The points labelled by roman numerals will be discussed below. (b) Number of detected singly ionized molecules, $\mathrm{CS}_{2}^{+}$(blue curve), and the doubly ionized molecules $\mathrm{CS}_{2}^{++}$(red curve) as a function of laser intensity. The statistical error bars are smaller than the symbols. To account for small pressure fluctuations, the raw counts are normalized to the average pressure for the corresponding data set. The black curve is a model for a seven-photon multiphoton ionization process that was used to fit the data (see FLMS experiment and data analysis in Methods section). Arb., arbitrary.

All of the data points in the case of the $200 \mathrm{fs}$ pulse have an intensity below $10^{13} \mathrm{~W} \mathrm{~cm}^{-2}$. For the case of alignment with shorter laser pulses $(60 \mathrm{fs})$, the alignment reaches a saturation value at a fluence of $0.48 \mathrm{~J} \mathrm{~cm}^{-2}$, which corresponds to an intensity of $8 \times 10^{12} \mathrm{~W} \mathrm{~cm}^{-2}$. Note that while the fraction of ionized molecules increases from less than a per cent at point III $\left(8 \times 10^{12} \mathrm{~W} \mathrm{~cm}^{-2}\right)$ to fully ionized at point $\mathrm{V}$ $\left(2.4 \times 10^{13} \mathrm{~W} \mathrm{~cm}^{-2}\right)$, the degree of alignment remains relatively constant over this interval. While ionization could be affecting the alignment, the fact that the alignment does not decrease with increasing ionization suggests that other mechanisms are at play. For example, structural changes, which change the moment of inertia of the molecule, can have a significant impact on alignment.

From diffraction pattern to molecular images. We employ the diffraction-difference method: A reference pattern is subtracted from each diffraction pattern to remove unwanted signals like experimental background, scattering from the buffer gas, and scattering from unexcited molecules $6,28,29$. The diffractiondifference pattern is thus due only to excited molecules. The term 'diffraction-difference pattern' in this manuscript represents the difference of a diffraction pattern and a reference pattern.

Figure 3a,b show the diffraction-difference pattern and its inverse Fourier transform corresponding to point I in Fig. 2a. The direction of the linear laser polarization is vertical. The reference pattern here is taken at a delay smaller than zero (the electron pulse arrives before the laser pulse), where the molecules are randomly oriented. The inverse Fourier transform in Fig. 3b at the positions corresponding to the $\mathrm{C}-\mathrm{S}$ and $\mathrm{S}-\mathrm{S}$ distances is positive in the vertical direction (along the laser polarization) and negative in the horizontal direction. This indicates that the number of molecules with their axis parallel to the laser polarization has increased, while the number of molecules in the perpendicular direction has decreased. In other words, molecules are aligned along the laser polarization direction.

When taking randomly oriented molecules as the reference (as we do by capturing the reference pattern at negative delay), the diffraction-difference pattern reflects the angular distribution of the whole molecular ensemble. The points I and III in Fig. 2 represent the fluences at which the degree of alignment saturates. By using those points as references, we can focus on molecular changes that appear for intensities beyond the saturation thresholds. We study three points after the saturation: point II for $200 \mathrm{fs}$ pulse excitation and points IV and $\mathrm{V}$ for $60 \mathrm{fs}$ excitation. These three points have slightly higher degrees of alignment than the reference points. When taking differences with these reference patterns, what remains are positive regions corresponding to molecules aligned with very narrow angular distributions and negative regions corresponding to broad angular distributions. This method circumvents the need for high alignment. Effectively, the difference gives us access to 3\% of molecules in the ensemble that are highly aligned. Structural changes can be measured with higher accuracy when the degree of alignment is high.

Figure $3 c, d$ show the diffraction-difference pattern between points II and point I and the corresponding inverse Fourier transform. The laser pulse intensities at points I and II are $4 \times 10^{12} \mathrm{~W} \mathrm{~cm}^{-2}$ and $7 \times 10^{12} \mathrm{~W} \mathrm{~cm}^{-2}$, respectively. The difference between the two points, II-I, yields a pattern corresponding to a very narrow angular distribution that approximates perfect alignment, as shown in Fig. 3c. This pattern results in an image of molecules that are highly aligned. Fig. $3 \mathrm{~d}$ shows a molecular image achieved using this method, where the position of the spots represents the distance between the atoms. With this method, the interatomic distances can be determined with a precision of $0.03 \AA$. The precision is limited by maximum diffraction angle recorded in the diffraction pattern and the signal-to-noise ratio in the data. The precision was determined by simulating diffraction patterns under the experimental conditions, varying the interatomic distance, and running the full data processing algorithm to determine the smallest change that can be reliably detected (see Interatomic distance determination and precision section in Methods section). The quoted precision is for the case where a single structure is measured. If there are excited states in addition to the ground state, the measurement will be an average of all the structures present in the sample.

Molecular image under low intensity. Figure 4a is an image of the molecule obtained from the diffraction-difference pattern between point II and point I, which corresponds to excitation with the $200 \mathrm{fs}$ laser pulse. For convenience, this image is referred to as 'data point II' in the following text. Figure $4 \mathrm{~b}$ shows the molecular image obtained by simulating a diffraction pattern from perfectly aligned molecules in their electronic ground state. The black curves show the expected C-S and S-S distances in the ground state (1.553 and $3.105 \AA$, respectively). The image in Fig. 4a corresponds to molecules that are confined within a cone with full-width at half-maximum (FWHM) of $9^{\circ}$ (see Degree of 
a

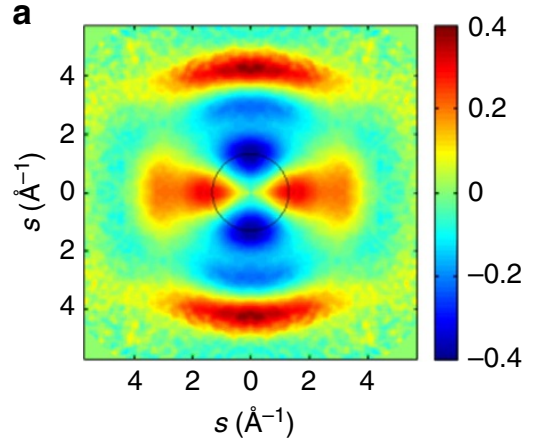

C

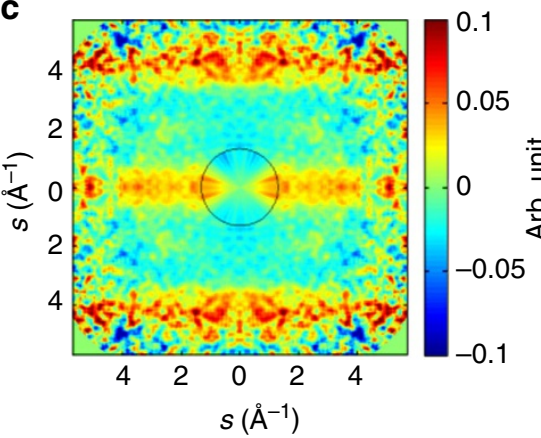

b

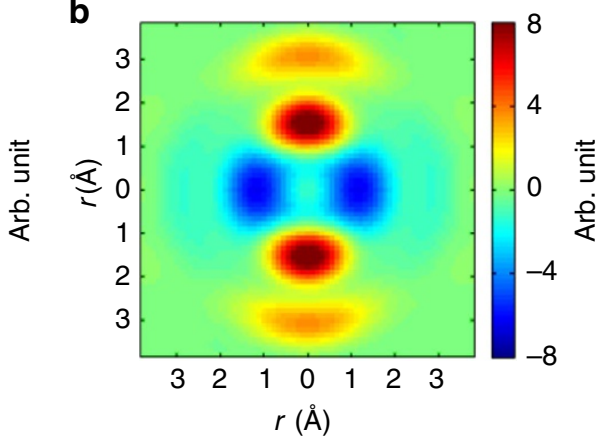

d

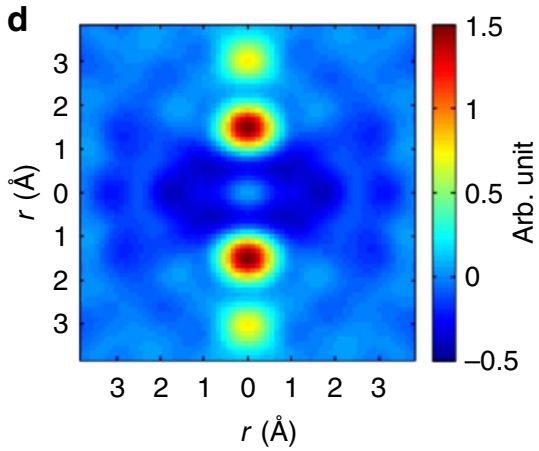

Figure 3 | From diffraction patterns to molecular images. (a) Diffraction-difference pattern of point I, reference pattern is taken at $t<0$; (b) inverse Fourier transform of $\mathbf{a}$; (c) diffraction-difference pattern between points II and I; (d) inverse Fourier transform of $\mathbf{c}$. The experimental data within the black circles in $\mathbf{a}$ and $\mathbf{c}$ are missing due to the beam stop, so these patterns are completed by letting the pixel values smoothly go to zero towards the centre. Arb., arbitrary.
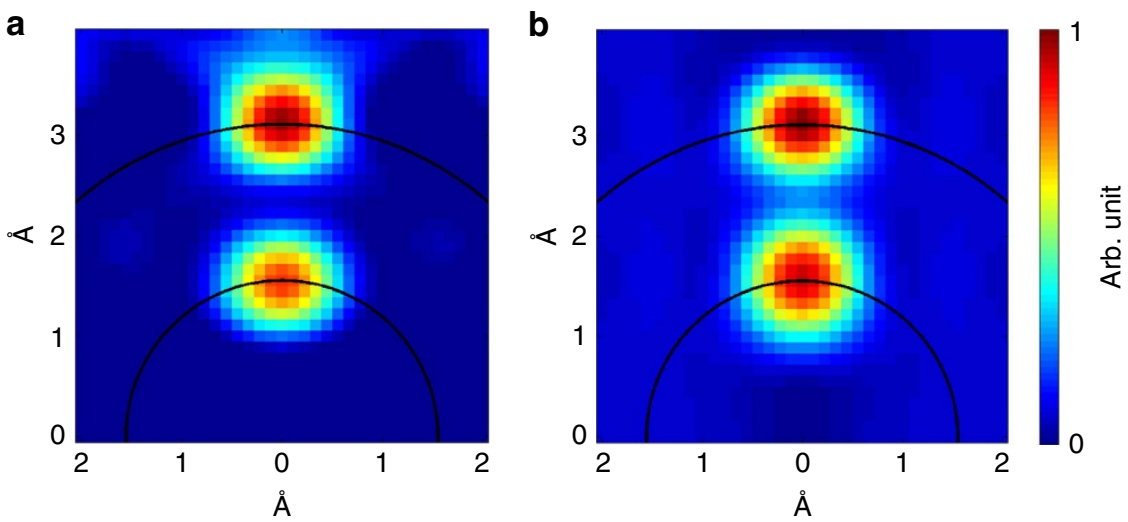

Figure 4 | Molecular images under low intensity. (a) Image of the $\mathrm{CS}_{2}$ molecule obtained from the diffraction-difference pattern between points 'II' $\left(7 \times 10^{12} \mathrm{~W} \mathrm{~cm}^{-2}\right)$ and ' I' $\left(4 \times 10^{12} \mathrm{~W} \mathrm{~cm}^{-2}\right)$. (b) Simulation of ground state, perfectly aligned molecules. The solid black curves show the C-S and S-S distances in the ground state for reference. The images are corrected for the finite size of the electron beam on the detector (see Correction for size of the electron beam section in Methods section). Arb., arbitrary.

alignment section in Methods section). For such a narrow distribution, the molecular image is very close to that obtained with perfect alignment.

The measured bond length is consistent with that of the electronic ground state. The two interatomic distances obtained from the data are $1.53 \pm 0.03 \AA$ and $3.11 \pm 0.03 \AA$ for the C-S and $\mathrm{S}-\mathrm{S}$ distances, respectively. The same data analysis performed on the simulated molecular image results in distances of $1.58 \pm 0.03 \AA$ and $3.13 \pm 0.03 \AA$. In both cases the distances are consistent with the bond lengths in the electronic ground state. The measured distances are displayed in Table 1. While the structure is consistent with the electronic ground state, the data does not rule out the excitation of vibrational modes or any excited state with bond lengths similar to the ground state. The excitation of vibration in addition to rotation could interfere with the alignment process, but our current experiment cannot detect small vibrations. The vibrational period would be shorter than the temporal resolution of our experiment, and the time-averaged bond distance would not change significantly. A bending vibration would result in a horizontal broadening of the CS spot. There is indeed some broadening in the CS spot in Fig. 4a, but the effect is not strong enough to draw any firm conclusions. Our method of molecular imaging is much more sensitive to changes in the position of the spots than to changes in width, so experiments with higher spatial resolution are needed to detect vibrations.

Molecular images under high intensity. Figure 5a shows the image of the molecules obtained from the diffraction-difference pattern between point IV $\left(1.3 \times 10^{13} \mathrm{~W} \mathrm{~cm}^{-2}\right)$ and point III 
Table 1 | Expected interatomic distances for ground state and interatomic distances extracted from simulated and measured diffraction patterns.

C-S distance ( $(\AA)$

Expected interatomic distances for the ground state

Ground state simulation

Data point ' $\mathrm{II}$ '

Data point 'IV'

Data point ' $\mathrm{V}$ '
1.553

$1.58 \pm 0.03$

$1.53 \pm 0.03$

$1.52 \pm 0.03$

$1.55 \pm 0.03$
S-S distance (A)

3.105

$3.13 \pm 0.03$

$3.11 \pm 0.03$

$3.27 \pm 0.03$

$3.31 \pm 0.03$
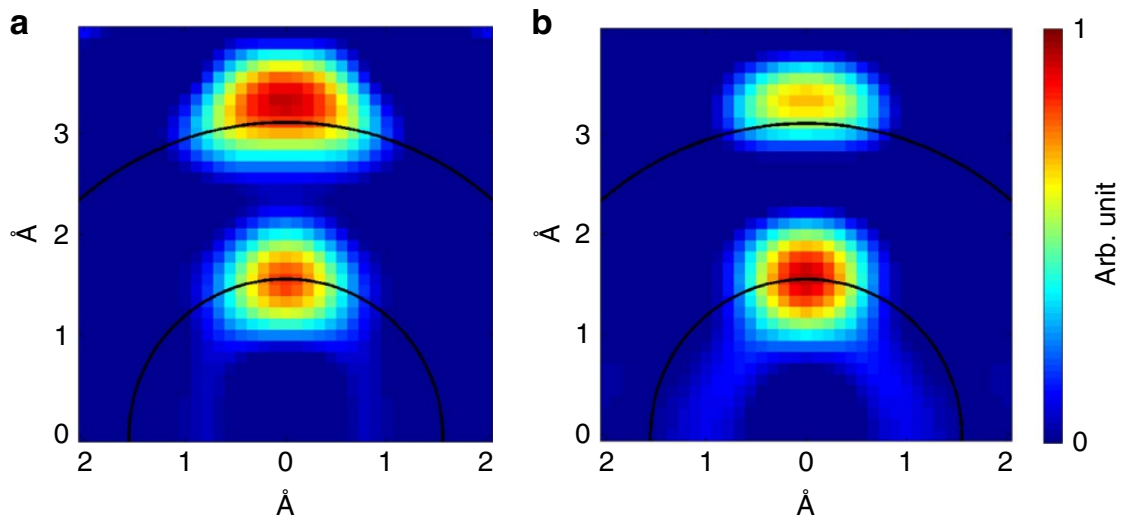

Figure 5 | Molecular images under high intensity. (a) Image of $\mathrm{CS}_{2}$ molecules taken from the diffraction-difference pattern between points 'IV' $\left(1.3 \times 10^{13} \mathrm{~W} \mathrm{~cm}^{-2}\right)$ and 'III' $\left(8 \times 10^{12} \mathrm{~W} \mathrm{~cm}^{-2}\right)$. (b) Image of $\mathrm{CS}_{2}$ molecules taken from the diffraction-difference pattern between points ' $\mathrm{V}$ ' $\left(2.4 \times 10^{13} \mathrm{~W} \mathrm{~cm}^{-2}\right)$ and 'III' $\left(8 \times 10^{12} \mathrm{~W} \mathrm{~cm}^{-2}\right)$. Arb., arbitrary.

$\left(8 \times 10^{12} \mathrm{~W} \mathrm{~cm}^{-2}\right)$, which corresponds to excitation with the $60 \mathrm{fs}$ laser pulse. Figure $5 \mathrm{~b}$ shows the image of the molecules obtained from the diffraction-difference pattern of point $\mathrm{V}\left(2.4 \times 10^{13} \mathrm{~W} \mathrm{~cm}^{-2}\right)$ with reference point III $\left(8 \times 10^{12}\right.$ $\mathrm{W} \mathrm{cm}-2)$. For convenience, these two images are named as 'data point IV' and 'data point $V$ ' in the following text. In data points IV and $\mathrm{V}$, the molecular ensemble has a lower degree of alignment than for data point II. The angular FWHM are measured to be $22^{\circ}$ and $23^{\circ}$, respectively. The S-S spot is visually broader in comparison to data point II, in which a $9^{\circ}$ angular FWHM was measured.

Both Fig. 5a,b are significantly different from the simulated ground state (Fig. $4 \mathrm{~b}$ ). In these two points, the S-S spots have moved upward, corresponding to a longer distance. Compared with the $\mathrm{S}-\mathrm{S}$ distance in the ground state, in Fig. 5a the distance has increased by $0.16 \AA$ while in Fig. $5 b$ the distance has increased by $0.20 \AA$. The measurements of interatomic distances from Fig. 5 are listed in Table 1. For the highest rotational state populated in our simulation $(J=57)$, the bond elongation due to centrifugal distortion is estimated to be at most $8 \times 10^{-4} \AA$ (refs 29,30) much lower than the measured bond lengthening. The longer bond length can be attributed to electronic excitation through a multiphoton process or to the formation of ions with longer bond lengths. There are multiple excited states that can be reached by multiphoton excitation, the majority of which have longer bond lengths ${ }^{31}$. One example is the excitation of the ${ }^{1} \mathrm{~B}_{2}\left({ }^{1} \Sigma_{u}^{+}\right)$state through the absorption of four photons with $800 \mathrm{~nm}$ wavelength. In this state the S-S distance is $0.13 \AA$ longer than in the ground state. We have also considered the possibility that the ionized molecule $\mathrm{CS}_{2}^{+}$would have a longer bond length. Simulations suggest that this is not the case ${ }^{32}$, however, further study of the ions is needed to completely rule out that possibility. At the highest intensity, there is a small fraction of $\mathrm{CS}_{2}^{++}$ions that might contribute to observing a longer bond length. It is likely that there are multiple excited states in the sample, and the measurement reflects the average structure of the different states. After being excited into a dissociative state, the molecule can be ionized via subsequent multiphoton absorption, or it can dissociate into neutral fragments.

The data present strong evidence for dissociation. In Fig. 5a,b, the ratio of the brightness of the $S-S$ spot to the brightness of the $\mathrm{C}-\mathrm{S}$ spot is reduced compared with the images in Fig. 4a,b. The effect is stronger in Fig. 5b, where the laser intensity is higher. Considering a single molecule, if one of the C-S bonds breaks, the spot corresponding to the $\mathrm{S}-\mathrm{S}$ distance in the molecular image vanishes as the $S$ atom is removed, while the intensity in the $C-S$ spot is reduced by a factor of two. Thus, when considering the whole ensemble, if a fraction of the molecules fragments, we expect that the intensity of the $\mathrm{S}-\mathrm{S}$ spot would decrease more than the intensity of the $\mathrm{C}-\mathrm{S}$ spot, in agreement with the measurement. Depending on the process, bond breaking can result in neutral or charged fragments. Neutral fragments are produced by electronic excitation into dissociative states, while charged fragments can be produced by bond breaking after ionization or by dissociation into neutral fragments that are then ionized by the laser. At the intensity corresponding to point $\mathrm{V}$ the fraction of charged fragments was measured with the FLMS setup to be $0.7 \%$. From the UEDAM results in Fig. $5 b$, we estimate that $2 / 3$ of the highly aligned molecules observed in the difference patterns have fragmented. Even though the difference-diffraction patterns captures only a fraction of all the molecules, it is clear that the number of charged fragments is too low to account for the large fraction of fragmented molecules. Therefore, the main mechanism for bond breaking is dissociation from electronically excited neutral molecules.

As can be seen in Fig. 5a,b, and in Table 1, while the distance of the S-S spot increases, the position of the C-S spot does not change and matches that of the ground state. This can be 
explained by considering all the contributions to the diffraction pattern and the temporal evolution of the excited states. The S-S spot results only from molecules that have not dissociated, while the C-S spot has contributions both from intact molecules and CS fragments. The CS fragment has a bond length of $1.5349 \AA$ (ref. 33), shorter than the ground state $\mathrm{CS}_{2}$. The dissociation happens on a fast time scale, leading to $\mathrm{CS}+\mathrm{S}$. For example, in the case of excitation to the ${ }^{1} \mathrm{~B}_{2}\left({ }^{1} \Sigma_{u}^{+}\right)$state where the dissociation time has been measured, a biexponential model with two decay channels was found to be a good fit for the dissociation ${ }^{7,9}$. The fast decay time constant was measured to be $<100 \mathrm{fs}$ and the slow decay time was between 500 and $800 \mathrm{fs}$. Comparing this with our temporal resolution of $1.0 \mathrm{ps}$, it is clear that the measurement is time averaged over the dissociation dynamics. The diffraction patterns were recorded at the time of maximum alignment, which were $760 \mathrm{fs}$ and $210 \mathrm{fs}$ after excitation for points IV and V, respectively. Thus, a significant fraction of the excited molecules will have already dissociated. While further experiments with improved temporal resolution are needed to fully characterize the dynamics, our current resolution has allowed us to capture a snapshot of the structure of the excited state. Our interpretation of the data is that for the alignment experiments with $200 \mathrm{fs}$ laser pulses the saturation of alignment is most likely caused by excitation of vibrational modes, since at this intensity level ionization is below $1 \%$ and no structural changes were observed. For the measurements with $60 \mathrm{fs}$ pulses, where the intensity is higher, the saturation of alignment is due to different mechanisms. Bond lengthening, dissociation and ionization are observed in this intensity range. We cannot separate the effect of ionization and structural changes on the alignment, but the fact that the degree of alignment remains relatively constant even as the degree of ionization increases by two orders of magnitude suggests that ionization is not the limiting mechanism. In the next section, we present additional evidence from the rotational dynamics that supports the previous evidence for dissociation.

Timing of the first alignment peak. The time delay for the molecules to reach the maximum degree of alignment after interacting with the laser pulse depends strongly on the moment of inertia of the molecule, as well as the laser pulse intensity. The peak alignment is defined as the maximum anisotropy in the pattern, as shown in Fig. 1b. If the $\mathrm{CS}_{2}$ molecule dissociates, the CS fragment, which has a lower moment of inertia, will reach its maximum alignment sooner. Figure 6 shows the time delay between the laser pulse and the first alignment peak as a function of fluence for (a) the $60 \mathrm{fs}$ laser pulse and (b) the $200 \mathrm{fs}$ laser pulse. In the case of the $200 \mathrm{fs}$ laser pulse, the time delay slowly
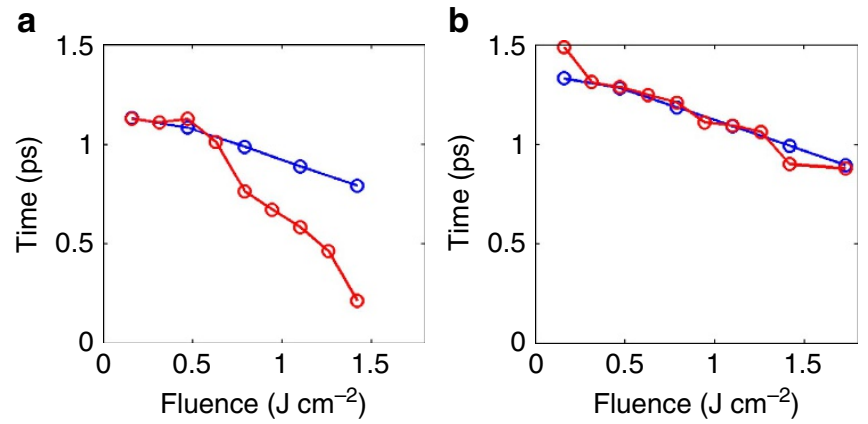

Figure 6 | Timing of the first alignment peak. Time delay between the laser and the peak alignment for (a) $60 \mathrm{fs}$ and (b) $200 \mathrm{fs}$. The blue and red curves represent simulated and experimental data, respectively. decreases with laser intensity, in good agreement with theory. This is also in good agreement with our previous conclusion that for the case of the longer pulses there are no significant structural changes (small vibrations would not significantly change the moment of inertia). For $60 \mathrm{fs}$ pulses where higher intensities are reached, experiment and theory agree for the lower fluence values but start to differ at a fluence of $0.7 \mathrm{~J} \mathrm{~cm}^{-2}$ (intensity $1.3 \times 10^{13} \mathrm{~W} \mathrm{~cm}^{-2}$ ), which corresponds to data point IV. This is the first point where we expect dissociation, and it also agrees with the saturation of the alignment in Fig. 2. The maximum alignment has contributions from all the molecules, only a fraction of which are dissociated, therefore, the moment of inertia cannot be directly retrieved from the measured time delay. However, the fact that the delay becomes significantly shorter than expected supports the conclusion that dissociation is significant at an intensity of $1.3 \times 10^{13} \mathrm{~W} \mathrm{~cm}^{-2}$.

\section{Discussion}

We have investigated the interaction of intense laser pulses with $\mathrm{CS}_{2}$ molecules and have observed saturation of alignment, deformation, dissociation and ionization. For excitation with long (200 fs) laser pulses, no structural changes are observed. The saturation of the alignment is most likely caused by the excitation of vibrational states. For higher intensities, multiphoton excitation leads to deformation, dissociation and ionization of the molecule. This electronic excitation also leads to saturation of the degree of alignment. Our conclusions are supported by spatially resolved molecular images, by the dynamics of the alignment process, and by independent measurements of the degree of ionization. Our results provide clear guidelines on intensity levels that can be used for impulsive alignment experiments without introducing structural changes, and show that the degree of alignment may reach a maximum value well below the threshold for multiphoton ionization. While the alignment saturates, there appears to be a range of intensities over which the structure does not change significantly, which can be a window for molecular imaging experiments. In molecular imaging experiments where impulsive alignment is used, it is important to perform careful measurements of the ionization, since a small increase in intensity can potentially result in a large increase of ionization. An alternative approach could be to use adiabatic alignment, where a long laser pulse is used to align the molecules, and the diffraction pattern is captured in the presence of the laser field ${ }^{34}$. Using a temporally shaped alignment laser pulse with slow ramp-up and rapid turn off ${ }^{35} \mathrm{can}$, in principle, achieve both higher degree of alignment and a fieldfree environment for diffraction. A similar study of electron diffraction under adiabatic alignment conditions would shed light on the intensity limits that can be used in that method and any additional effects that might result from performing the diffraction in the presence of a strong laser field. For future experiments, the temporal resolution of UEDAM could be improved to around $100 \mathrm{fs}$ using $\mathrm{keV}$ electron pulses compressed by an RF cavity ${ }^{36}$ in combination with tilted laser pulses $^{37,38}$ or by using $\mathrm{MeV}$ electron beams ${ }^{39}$. The spatial resolution can be improved by increasing the beam current, which will allow increasing the area of momentum space captured in the diffraction patterns.

\section{Methods}

UEDAM experiment. $\mathrm{CS}_{2}$ molecules are seeded into buffer gas of helium with a 1:20 ratio for rotational cooling. The de Laval nozzle has a $30 \mu \mathrm{m}$ diameter at the throat and a $90 \mu \mathrm{m}$ diameter at the exit. A supersonic jet is formed after the nozzle The nozzle backing pressure is set to 1,000 Torr. The sizes of electron beam, laser beam and gas jet are 100, 100 and $200 \mu \mathrm{m}$ FWHM, respectively. The $\mathrm{CS}_{2}$ gas density is $\sim 5 \times 10^{15} \mathrm{~cm}^{-3}$ in the interaction region, and the rotational temperature is estimated to be around $30 \mathrm{~K}$ (ref. 40). The electron pulses are
} 
generated using a photocathode and accelerated in a static field to a kinetic energy of $25 \mathrm{keV}$, which corresponds to $\lambda_{\mathrm{e}}=7.7 \mathrm{pm}$. The number of electrons per pulse is set to 1,000 to reduce the space-charge effect and maintain a pulse duration of $\sim 400 \mathrm{fs}$ at the sample. The overall instrument response function for this experiment, including the velocity mismatch between photons and electrons, is $1.0 \mathrm{ps}$. This is sufficient to capture diffraction patterns, since the lifetime of the alignment is $\sim 2 \mathrm{ps}$. The laser generates pulses with $2 \mathrm{~mJ}$ energy and a wavelength of $800 \mathrm{~nm}$ at a repetition rate of $5 \mathrm{kHz}$.

FLMS experiment and data analysis. The ions are collected using a mass spectrometer that has a fixed, micrometer-sized interaction volume that is smaller than the focal volume of the laser, thus avoiding volume averaging. The number of ions was measured to be proportional to the gas pressure, which indicates that space-charge effects were not significant. For the singly ionized $\mathrm{CS}_{2}^{+}$ions, the number of measured ions increases with the seventh power of the laser intensity, as expected for a seven photon-ionization process, for an intensity up to $1.2 \times$ $10^{13} \mathrm{~W} \mathrm{~cm}^{-2}$. The data points for intensities below $1.1 \times 10^{13} \mathrm{~W} \mathrm{~cm}^{-2}$ were used to fit to a theoretical model for the ionization probability for seven-photon multiphoton ionization $P(I)=1-\exp \left[-\left(I / I_{\text {sat }}\right)^{7}\right]$, where $P$ is the probability, $I$ is the laser pulse intensity and $I_{\text {sat }}$, the saturation intensity, is the fit parameter. The fit resulted in a value of $I_{\text {sat }}=1.85 \times 10^{13} \mathrm{~W} \mathrm{~cm}^{-2}$. The model and the data agree very well for intensities below $1.2 \times 10^{13} \mathrm{~W} \mathrm{~cm}^{-2}$, and for higher values the data underestimate the number of ions compared with the model.

The $100 \%$ value for the model was taken to be equal to the maximum number of total ions measured experimentally (including both singly and doubly ionized molecules). This means that the measurement provides a lower estimate for the ionization and the model provides an upper estimate, since the maximum number of measured ions could be below $100 \%$.

Molecular images. All diffraction patterns are normalized to the scattering from individual atoms: $I=I_{\mathrm{raw}} / I_{\mathrm{at}}$, where $I$ represents the normalized diffraction pattern, $I_{\text {raw }}$ is the raw diffraction pattern, and $I_{\text {at }}$ is the sum of the scattering intensity of individual atoms.

In this work, anisotropy is used to trace the evolution of alignment. Anisotropy is defined as the difference in total counts between the horizontal and the vertical quadrants in the range of $s=1-3.5 \AA^{-1}$ (Fig. 1b) in the normalized diffraction pattern. The quantity $s=\frac{4 \pi}{2} \sin (\theta / 2)$ is the momentum change of the scattered electrons, where $\lambda_{\mathrm{e}}$ is the êlectron wavelength and $\theta$ is the scattering angle.

The inverse Fourier transform of the diffraction pattern intensity (without phase) of a single molecule gives the autocorrelation function of the molecular structure. A diffraction pattern of a molecular ensemble is an incoherent sum of diffraction patterns from each molecule:

$$
\mathcal{F}^{-1}\left[\sum_{\mathrm{i}}\left|\mathcal{F}\left[a_{\mathrm{i}}\right]\right|^{2}\right]=\sum_{\mathrm{i}} a_{\mathrm{i}} * a_{\mathrm{i}}
$$

In this equation, the left side is an inverse Fourier transform of the incoherent sum of many single-molecule diffraction patterns, and the right side is the sum of the autocorrelation functions of all molecules.

Correction for the size of the electron beam. The electron beam is focused onto the gas jet, and the size of the beam increases as it propagates after the gas jet. Thus, the finite size of the electron beam on the detector will reduce the sharpness of diffraction patterns. The diffraction pattern measured experimentally is a convolution of the ideal diffraction pattern with the measured two-dimensional shape of the electron beam on the detector. This is equivalent to multiplying the autocorrelation functions by the inverse Fourier transform of the electron beam. Therefore, this effect is corrected by dividing the autocorrelation functions by an envelope that is the inverse Fourier transform of the electron beam.

Interatomic distance determination and precision. The interatomic distances are determined by the Gaussian approximation sub-pixel peak position determination algorithm ${ }^{41}$. The $0.03 \AA$ precision is calculated by applying this algorithm to simulated patterns with 50 different bond lengths and taking the s.d. of the difference between output lengths and input lengths. In our experiment, the spatial resolution is given by $\delta=2 \pi / s_{\max } \approx 1.2 \AA$, which gives the width of the measured spots in the molecular images. The precision in the interatomic distances is given by the position of the spots, which can be determined to higher accuracy than the width of the spots, as long as they are well separated. As a comparison, static gas-phase electron diffraction experiments ${ }^{42}$ typically achieve a precision of $0.001 \AA$ with a resolution of $\delta \approx 0.2 \AA$.

Degree of alignment. The degree of alignment is usually measured by the quantity $<\cos ^{2} \alpha>$. However, this metric is not applicable to the case of differencediffraction patterns. In this case the total population will sum up to zero. The molecular images in this work contain a narrow positive population section and a broad negative population section. The positive population constructs a cone shape. We use the FWHM of the full-opening angle of the positive cone to characterize the degree of alignment. The angular distribution is extracted using the pBasex Abel inversion algorithm ${ }^{43}$. The finite scattering angle captured in the diffraction patterns, corresponding to a convolution with a Gaussian envelope in the molecular image, is taken into account in the algorithm.

Alignment simulation. The simulation of impulsive alignment is calculated using a linear rigid rotor interacting with a non-resonant pulse described by the time-dependent Schrodinger equation ${ }^{44}$. To better match the experimental conditions, we have also simulated the effect of a pre-pulse and a pedestal with up to $1 \%$ of the peak intensity and 20 times longer pulse duration. Neither of these significantly changed the maximum degree of alignment.

Diffraction pattern simulation. The simulations of diffraction patterns are calculated using cylindrical harmonics ${ }^{45}$. In the simulations, the atoms are assumed to be stationary at their equilibrium positions without vibrations.

\section{References}

1. Spence, J. C. H. et al. Diffraction and imaging from a beam of laser-aligned proteins: resolution limits. Acta Crystallogr. A 61, 237-245 (2005).

2. Ho, P. J. et al. Molecular structure determination from $\mathrm{x}$-ray scattering patterns of laser-aligned symmetric-top molecules. J. Chem. Phys. 131, 131101 (2009).

3. Saldin, D. K. et al. New light on disordered ensembles: Ab initio structure determination of one particle from scattering fluctuations of many copies. Phys. Rev. Lett. 106, 105501 (2011).

4. Reckenthaeler, P. et al. Time-resolved electron diffraction from selectively aligned molecules. Phys. Rev. Lett. 102, 213001 (2009).

5. Küpper, J. et al. X-ray diffraction from isolated and strongly aligned gas-phase molecules with a free-electron laser. Phys. Rev. Lett. 112, 83002 (2014).

6. Hensley, C. J., Yang, J. \& Centurion, M. Imaging of isolated molecules with ultrafast electron pulses. Phys. Rev. Lett. 109, 133202 (2012).

7. Bisgaard, C. Z. et al. Time-resolved molecular frame dynamics of fixed-in-space $\mathrm{CS}_{2}$ molecules. Science 323, 1464-1468 (2009).

8. Hockett, P., Bisgaard, C. Z., Clarkin, O. J. \& Stolow, A. Time-resolved imaging of purely valence-electron dynamics during a chemical reaction. Nat. Phys. 7, 612-615 (2011).

9. Townsend, D. et al. ${ }^{1} \mathrm{~B}_{2}\left({ }^{1} \Sigma_{\mathrm{u}}^{+}\right)$excited state decay dynamics in $\mathrm{CS}_{2}$. J. Chem. Phys. 125, 234302 (2006).

10. Wang, K., McKoy, V., Hockett, P. \& Schuurman, M. S. Time-resolved photoelectron spectra of $\mathrm{CS}_{2}$ : dynamics at conical intersections. Phys. Rev. Lett. 112, 113007 (2014).

11. Fuji, T., Suzuki, Y. I., Horio, T. \& Suzuki, T. Excited-state dynamics of $\mathrm{CS}_{2}$ studied by photoelectron imaging with a time resolution of $22 \mathrm{fs}$. Chem. Asian J. 6, 3028-3034 (2011).

12. Kumarappan, V. et al. Multiphoton electron angular distributions from laser-aligned $\mathrm{CS}_{2}$ molecules. Phys. Rev. Lett. 100, 93006 (2008).

13. Kumar, G. R., Safvan, C. P., Rajgara, F. A. \& Mathur, D. Intense laser field ionisation of $\mathrm{CS}_{2}$ at $532 \mathrm{~nm}$. Does dissociation precede ionisation? Chem. Phys. Lett. 217, 626-630 (1994).

14. Banerjee, S., Kumar, G. R. \& Mathur, D. Intensity-selective, field-induced dissociative ionization of $\mathrm{CS}_{2}$ by femtosecond-duration light pulses. J. Phys. B At. Mol. Opt. Phys. 32, 4277-4292 (1999).

15. Mathur, D. et al. Angular distributions of the products of dissociative ionization of molecules by intense laser fields. Laser Phys. 7, 829-838 (1997).

16. Iwasaki, A., Hishikawa, A. \& Yamanouchi, K. Real-time probing of alignment and structural deformation of $\mathrm{CS}_{2}$ in intense nanosecond laser fields. Chem. Phys. Lett. 346, 379-386 (2001).

17. Vallance, C. 'Molecular photography': velocity-map imaging of chemical events. Philos. Trans. A Math. Phys. Eng. Sci. 362, 2591-2609 (2004).

18. Ashfold, M. N. R. et al. Imaging the dynamics of gas phase reactions. Phys. Chem. Chem. Phys. 8, 26-53 (2006).

19. Neutze, R., Wouts, R., van der Spoel, D., Weckert, E. \& Hajdu, J. Potential for biomolecular imaging with femtosecond X-ray pulses. Nature 406, 752-757 (2000).

20. Srinivasan, R., Feenstra, J. S., Park, S. T., Xu, S. \& Zewail, A. H. Dark structures in molecular radiationless transitions determined by ultrafast diffraction. Science 307, 558-563 (2005).

21. Centurion, M., Reckenthaeler, P., Krausz, F. \& Fill, E. Picosecond electron diffraction from molecules aligned by dissociation. J. Mol. Struct. 978, 141-146 (2010).

22. Strohaber, J. \& Uiterwaal, C. J. G. J. In situ measurement of three-dimensional ion densities in focused femtosecond pulses. Phys. Rev. Lett. 100, 23002 (2008).

23. Scarborough, T. D., Strohaber, J., Foote, D. B., McAcy, C. J. \& Uiterwaal, C. J. G. J. Ultrafast REMPI in benzene and the monohalobenzenes without the focal volume effect. Phys. Chem. Chem. Phys. 13, 13783-13790 (2011).

24. Scarborough, T. D., Foote, D. B. \& Uiterwaal, C. J. G. J. Ultrafast resonanceenhanced multiphoton ionization in the azabenzenes: pyridine, pyridazine, pyrimidine, and pyrazine. J. Chem. Phys. 136, 54309 (2012). 
25. Hertel, I. V. \& Schulz, C.-P. Atoms, Molecules and Optical Physics 2: Molecules and Photons-Spectroscopy and Collisions (Springer-Verlag Berlin Heidelberg, 2015).

26. Holmegaard, L. et al. Control of rotational wave-packet dynamics in asymmetric top molecules. Phys. Rev. A 75, 051403 (2007).

27. Centurion, M., Reckenthaeler, P., Trushin, S. A., Krausz, F. \& Fill, E. E. Picosecond electron deflectometry of optical-field ionized plasmas. Nat. Photonics 2, 315-318 (2008).

28. Srinivasan, R., Lobastov, V. A., Ruan, C.-Y. \& Zewail, A. H. Ultrafast electron diffraction (UED): a new development for the 4D determination of transient molecular structures. Helv. Chim. Acta 86, 1763-1838 (2003).

29. Toyama, M., Oka, T. \& Morino, Y. Effect of vibration and rotation on the internuclear distance. J. Mol. Spectrosc. 13, 193-213 (1964).

30. Kummli, D. S., Frey, H. M. \& Leutwyler, S. Femtosecond degenerate four-wave mixing of carbon disulfide: High-accuracy rotational constants. J. Chem. Phys. 124, 144307 (2006)

31. Zhang, Q. \& Vaccaro, P. H. Ab initio studies of electronically excited carbon disulfide. J. Phys. Chem. 99, 1799-1813 (1995).

32. Hochlaf, M. Ab initio investigations of the unimolecular decay of $\mathrm{CS}_{2}^{+}$for internal energies lower than $10 \mathrm{eV}$. J. Phys. B At. Mol. Opt. Phys. 37, 595-608 (2004).

33. Mockler, R. C. \& Bird, G. R. Microwave spectrum of carbon monsulfide. Phys. Rev. 98, 1837-1839 (1955).

34. Hoshina, K., Yamanouchi, K., Ohshima, T., Ose, Y. \& Todokoro, H. Direct observation of molecular alignment in an intense laser field by pulsed gas electron diffraction II: analysis of anisotropic diffraction image. Chem. Phys. Lett. 353, 33-39 (2002).

35. Seideman, T. On the dynamics of rotationally broad, spatially aligned wave packets. J. Chem. Phys. 115, 5965-5973 (2001).

36. Van Oudheusden, T. et al. Compression of subrelativistic space-chargedominated electron bunches for single-shot femtosecond electron diffraction. Phys. Rev. Lett. 105, 264801 (2010).

37. Zhang, P., Yang, J. \& Centurion, M. Tilted femtosecond pulses for velocity matching in gas-phase ultrafast electron diffraction. New. J. Phys. 16, 083008 (2014).

38. Danielius, R. et al. Matching of group velocities by spatial walk-off in collinear three-wave interaction with tilted pulses. Opt. Lett. 21, 973-975 (1996).

39. Yang, J. et al. 100-femtosecond $\mathrm{MeV}$ electron source for ultrafast electron diffraction. Radiat. Phys. Chem. 78, 1106-1111 (2009).

40. Pullman, D. R., Friedrich, B. \& Herschbach, D. R. Facile alignment of molecular rotation in supersonic beams. J. Chem. Phys. 93, 3224-3236 (1990).
41. Naidu, D. K. \& Fisher, R. B. A comparative analysis of algorithms for determining the peak position of a stripe to sub-pixel accuracy. Proc. Br. Mach. Vis. Conf. 1991, 28.1-28.9 (1991).

42. Huntley, C. M., Rankin, D. W. H. \& Buildings, K. Gas-phase molecular structure of bis(difluorophosphino)amine, determined by electron diffraction. J. Chem. Soc. Dalt. Trans. 6, 954-957 (1980).

43. Garcia, G. A., Nahon, L. \& Powis, I. Two-dimensional charged particle image inversion using a polar basis function expansion. Rev. Sci. Instrum. 75, 4989-4996 (2004).

44. Seideman, T. \& Hamilton, E. Nonadiabatic alignment by intense pulses. Concepts, theory, and directions. Adv. At. Mol. Opt. Phys. 52, 289-329 (2005).

45. Saldin, D. K., Shneerson, V. L., Starodub, D. \& Spence, J. C. H. Reconstruction from a single diffraction pattern of azimuthally projected electron density of molecules aligned parallel to a single axis. Acta Crystallogr. A 66, 32-37 (2010).

\section{Acknowledgements}

J.Y. and M.C. were supported by the US Department of Energy Office of Science, Office of Basic Energy Sciences under Award Number DE-SC0003931. J.B. and C.J.U. were partially supported by US Department of Education GAANN grants numbers P200A090156 and P200A120188 and by the National Science Foundation EPSCoR RI Track-2 CA Award No. IIA-1430519 (Cooperative Nebraska-Kansas grant). We thank Christopher Hensley for his contribution to the initial setup of this experiment.

\section{Author contributions}

M.C. designed the UEDAM experiments; J.Y. performed the UEDAM experiments; M.C and J.Y. developed data analysis methods; J.Y. analysed UEDAM experimental data; J.Y. simulated alignment process and diffraction patterns; J.B. performed the FLMS measurements, J.B. and C.J.U. analysed the FLMS data. M.C. and J.Y. wrote the manuscript; M.C. supervised the project.

\section{Additional information}

Competing financial interests: The authors declare no competing financial interests.

Reprints and permission information is available online at http://npg.nature.com/ reprintsandpermissions/

How to cite this article: Yang, J. et al. Imaging of alignment and structural changes of carbon disulfide molecules using ultrafast electron diffraction. Nat. Commun. 6:8172 doi: $10.1038 /$ ncomms9172 (2015). 